\title{
Increased frequency of detection of Chlamydophila pneumoniae in asthma
}

\author{
G.L. Biscione*, J. Corne*, A.J. Chauhan*, S.L. Johnston*\#
}

Increased frequency of detection of Chlamydophila pneumoniae in asthma. G.L. Biscione, J. Corne, A.J. Chauhan, S.L. Johnston. (C) ERS Journals Ltd 2004.

ABSTRACT: Previous studies have suggested that chronic Chlamydophila pneumoniae infection may play a role in the pathogenesis of asthma. However, most studies have been based on serology and have been unable to differentiate acute from chronic infection.

The present authors assessed the presence of acute and chronic $C$. pneumoniae infection in 74 spouse pairs, each consisting of one atopic asthmatic and one nonatopic nonasthmatic. Nasal secretions were sampled every 2 weeks from October to December and actively replicating $C$. pneumoniae infection was detected by specific RT-PCR.

$C$. pneumoniae was detected in 31 out of 709 samples analysed, $23(6.4 \%)$ were positive in 362 samples from asthmatic participants and in eight out of $347(2.3 \%)$ samples from their normal spouses (with a significant difference in infection rates, $95 \%$ confidence interval: $4.2 \%, 1.2-7.2 \%)$. A total of $16(22 \%)$ asthmatic and seven $(9 \%)$ normal participants were positive at least once during the study.

These data confirm that Chlamydophila pneumoniae infection is detected more frequently among asthmatic participants than normal control participants. Further studies are required to confirm whether infections are also present in the lower airway and whether Chlamydophila pneumoniae infection plays a role in disease pathogenesis. Eur Respir J 2004; 24: 745-749.
*University Medicine, Southampton General Hospital, Southampton, and "Dept of Respiratory Medicine, National Heart and Lung Institute, Imperial College London, London, UK.

Correspondence: G.L. Biscione, Centre of Pulmonary Medicine and Rehabilitation, S. Raffaele Hospital (Tosinvest Sanitá), Via Dei Laghi km 19, 600, 00049-Velletri, Rome, Italy. Fax: 39069636715

E-mail: dr_biscione@yahoo.it

Keywords: Asthma, Chlamydophila pneumoniae, RT-PCR

Received: April 262004

Accepted after revision: August 152004

This study received funding from the National Asthma Campaign (Grant 201 to S.L. Johnson) and both J. Corne and A.J. Chauhan were Medical Research Council, UK Clinical Training Fellows.
Asthma is an important chronic respiratory disorder responsible for a heavy burden of illness [1]. Recent studies demonstrate that acute respiratory viral infections, principally rhinovirus, are associated with the majority of asthma exacerbations in adults and children [2,3].

Chlamydophila pneumoniae is a respiratory pathogen known to infect the upper and lower respiratory tracts [4], and although many infections are believed to be asymptomatic, it is also known to cause a variety of respiratory syndromes [5-8]. Recent studies have suggested that this organism may have a role in the pathogenesis of newly diagnosed and chronic stable asthma in both adults and children [9-13]. However, these studies have been based on serological tests, which cannot reliably differentiate between past and present infection or acute and chronic infection and which may be cross-reactive with other Chlamydia species.

Further evidence suggesting a role for chronic $C$. pneumoniae infection in asthma pathogenesis comes from a recent study that demonstrated improvement in asthma severity after 6 weeks of treatment with a macrolide antibiotic, active against $C$. pneumoniae, in asthmatic participants seropositive for $C$. pneumoniae [14]. However, the improvement was small and only apparent during continued therapy, and the study was unable to differentiate anti-inflammatory properties of the macrolide from antimicrobial properties. Furthermore, the study by BLACK et al. [14] did not look for direct evidence of infection at recruitment, evidence of eradication of infection, or investigate whether deterioration following cessation of therapy was related to re-infection [15].

The present authors have previously used PCR to demonstrate a high prevalence of $C$. pneumoniae infection in nasal aspirates (NA) from asthmatic children and a relationship between the local specific-immunoglobulin (Ig)A immune response and asthma severity [16]. These data suggested that chronic low-grade $C$. pneumoniae infection may be implicated in asthma pathogenesis. However, this study did not include a control group of normal children. Furthermore, no previous study has included regular sampling to determine duration of detection.

The present authors have recently shown that asthmatic subjects are more susceptible to rhinovirus infections than normal individuals [17] and that asthmatic subjects have relatively impaired antiviral (interferon (IFN)- $\gamma$ and interleukin-12 production) immunity to rhinovirus infection [18]. IFN- $\gamma$ production is known to be important in the resolution of $C$. pneumoniae infection $[19,20]$. The current authors, therefore, hypothesised that asthmatic individuals may be more susceptible to $C$. pneumoniae infection than normal individuals.

The aim of the present study was to investigate the frequency of $C$. pneumoniae infections in participants with atopic asthma and in control participants without asthma or atopic disease using direct detection by PCR. Regular sampling was carried out to allow the current authors to determine duration of any positive detection. 


\section{Methods}

\section{Participant recruitment and sample collection}

Spouse pairs consisting of an atopic asthmatic individual and a nonatopic nonasthmatic partner, both aged between 18-50 yrs, were recruited to the study. The diagnosis of asthma was confirmed by use of a modified International Union against Tuberculosis and Lung Disease questionnaire [21], and atopic status was confirmed by skin prick testing to a range of common allergens. All participants were nonsmoking and asthmatic participants were mild-to-moderate in severity, being treated in accordance with steps 1-3 of the British Thoracic Society guidelines. Treatment with oral steroids was an exclusion criterion. The spouse-pair study design was adopted to ensure that the exposure to infectious organisms would be as closely matched as possible, as exposure is a major potential confounding variable. Full details of recruitment methods are given in a previous report [17].

Participants attended the department every 2 weeks with each member of a couple attending on the same day, or within $24 \mathrm{~h}$ if this was not possible. At each visit a NA was taken as previously reported [17]. A total of 74 spouse pairs entered the study and provided a total of 709 NA samples that were analysed for the presence of $C$. pneumoniae using RT-PCR. All participants gave signed written informed consent and the study was approved by the Southampton University and Hospitals Joint Ethics Committee, Southmapton, UK.

\section{Chlamydophila pneumoniae-specific $R T$-PCR}

A nested RT-PCR assay for replicating C. pneumoniae was performed on cDNA synthesised with random-hexamers from total RNA extracted from the NA samples. PCR was carried out to detect mRNA of the major outer membrane protein (MOMP) gene. This PCR was developed from a previous PCR that used DNA amplification only [16] and which may, therefore, have detected nonreplicating chlamydophilal DNA. The MOMP is produced in abundance during chlamydophilal replication. Therefore, detection of mRNA coding for this protein would indicate active replication. Following pre-incubation at $94^{\circ} \mathrm{C}$ for $2 \mathrm{~min}$, the reaction cycle consisted of denaturation at $94^{\circ} \mathrm{C}$ for $20 \mathrm{~s}$, annealing at $52^{\circ} \mathrm{C}$ for $20 \mathrm{~s}$ for the first round or $63^{\circ} \mathrm{C}$ for $20 \mathrm{~s}$ for the second round, and the extension at $72^{\circ} \mathrm{C}$ for $30 \mathrm{~s}$, with 25 cycles for the first round and 35 cycles for the second round. The sensitivity by gel electrophoresis (GE) of the secondround product was $4.5 \mathrm{fg}$ of $C$. pneumoniae cDNA. All positive samples were reconfirmed by repeat analysis and all RNA extractions, primary PCR amplifications and secondary PCR amplifications were undertaken in dedicated laboratory areas with dedicated pipettes and filtered tips. A minimum of two negative controls were incorporated into each run.

The outer (first round) primers used were APNOU (5' AAT TCT CTG TAA ACA AAC CC 3') and APNOL (5' ATT AAG AAG CTC TGA GCA TA $3^{\prime}$ ), and the inner (second round) primers were APN1 (5' AGC CTA ACA TGT ACA CTC TGA T $3^{\prime}$ ) and APN2 (5' TGC CAA CAG ACG CTG GCG T $3^{\prime}$ ) generating a 487 base-pair product detected by GE.

A short cloned fragment of $C$. pneumoniae DNA and two clinical samples known to be positive for $C$. pneumoniae from a previous study [16] were used for positive controls.
Negative controls were noninfected PBS. The sensitivity by $\mathrm{GE}$ of the second round product was $4.5 \mathrm{fg}$ of $C$. pneumoniae DNA.

\section{Analysis of data}

Within the 709 samples, the association between $C$. pneumoniae infection and asthma was investigated using Pearson's Chi-squared test. To allow for the fact that some individuals were infected on more than one occasion, and that atopic asthmatic and normal partners were paired with respect to exposure to infectious agents (in addition to the previously described positive sample analysis), a paired spouse analysis on frequency of $C$. pneumoniae infection among couples occurring at any time during the 3-month study was performed using the Wilcoxon signed-rank test. The association among asthmatic participants of the use of inhaled steroids with one or more $C$. pneumoniae infections was analysed using Fisher's exact test.

\section{Results}

\section{Baseline characteristics}

A total of 74 couples completed the study. The median age of both groups was 36 yrs (range 26-50 yrs for the asthmatic group and 26-47 yrs for the nonasthmatic group). A total of 709 NA were analysed.

\section{Chlamydophila pneumoniae detection}

There were a total of $31 \mathrm{C}$. pneumoniae positive samples, of which 23 out of $362(6.4 \%)$ were from the atopic asthmatic participants and eight out of $347(2.3 \%)$ were from the normal spouses (Pearson's Chi-squared: 7.284, $\mathrm{p}=0.007$; difference $4.2 \%, 95 \%$ CI: $1.2-7.2 \%$ ) (fig. 1a). All positive samples gave unequivocally positive bands on RT-PCR (fig. 2).

The paired analysis confirmed a significantly higher frequency of $C$. pneumoniae detections in the asthmatic group (16 out of $74(22 \%)$ infected on one or more occasion during the study) compared with the normal control group (seven out of $74(9 \%)$ infected; $\mathrm{p}=0.012$ using the Wilcoxon signed-rank test; fig. 1b).

\section{Relationship between Chlamydophila pneumoniae and inhaled steroid therapy}

An association between $C$. pneumoniae infection and disease has been suggested in participants with steroiddependent asthma $[11,22]$. For this reason, an association between the use of inhaled steroids and the presence of one or more $C$. pneumoniae infection was only examined in asthmatic participants. A total of 52 out of 74 atopic asthmatic participants were treated with inhaled steroids $(70 \%)$, while $22(30 \%)$ were not. C. pneumoniae was detected in 10 out of $52(19 \%)$ of the participants treated and six out of $22(27 \%)$ not treated with inhaled steroids. The difference was not significant using Fisher's exact test $(\mathrm{p}=0.539)$.

\section{Acute versus chronic Chlamydophila pneumoniae infection}

Of 31 positive samples, 24 were preceded by a negative sample taken 14 days earlier and followed by a negative 

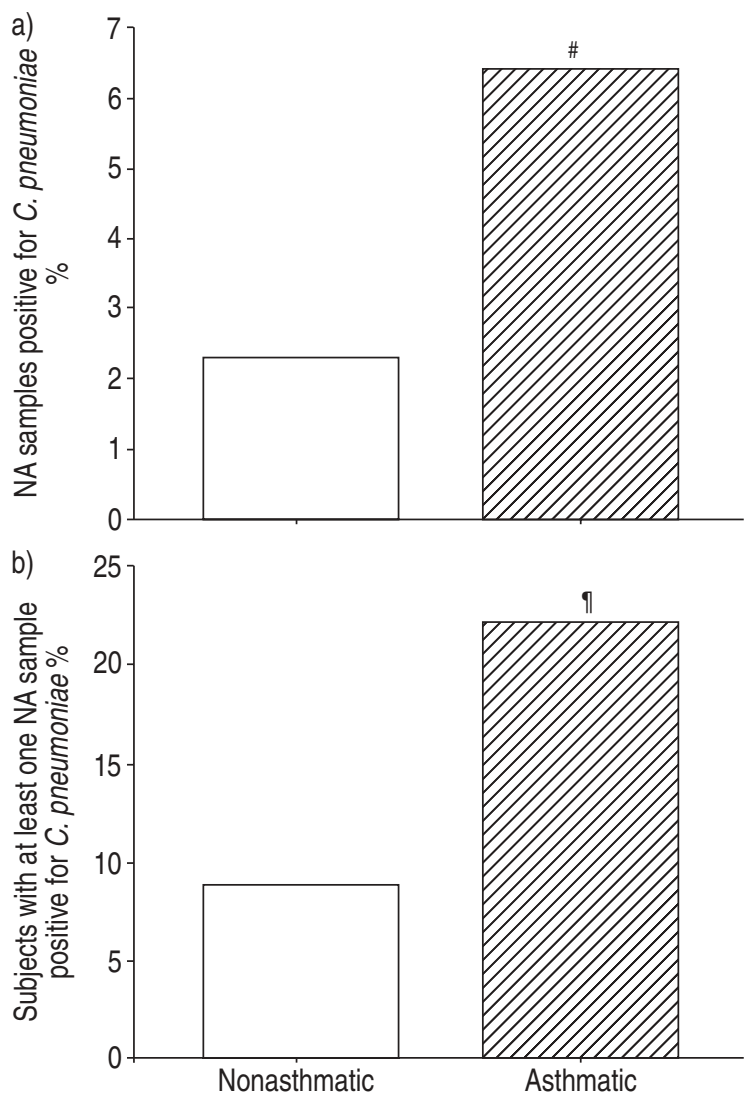

Fig. 1.- Frequency of detection of Chlamydophila pneumoniae mRNA for nonatopic nonasthmatic and atopic asthmatic subjects. a) Percentage of total nasal aspirate (NA) samples collected, positive for detection of $C$. pneumoniae mRNA, and b) paired analysis of percentage of subjects with at least one NA sample positive for detection of $C$. pneumoniae mRNA at any time during the 3-month study period for nonatopic nonasthmatic $(\square)$ and atopic asthmatic $(\mathbb{Z})$ subjects. ${ }^{\#}$ : $\mathrm{p}=0.007$, Pearson's Chi-squared test; : $\mathrm{p}=0.012$, Wilcoxon signed-rank test.

sample taken 14 days later, suggesting acute infections of $<28$ days duration. There were three participants in whom consecutive samples were positive. One participant was positive for two consecutive samples, having been previously negative, and became negative on the next sample; the maximum duration of this infection, therefore, was $<42$ days. In the remaining two, the duration could not be defined as one was positive at entry to the study and the other was positive at the end of the study. The participant positive at entry was positive for three consecutive samples, but then became negative on all subsequent samples. The participant positive at the end of the study was negative initially and then positive for two consecutive samples at the end of the study.
All three participants with consecutive positive samples were asthmatic.

\section{Discussion}

The authors of the present study have shown a higher frequency of detection of $C$. pneumoniae in NA samples from atopic asthmatic participants compared to their nonatopic nonasthmatic spouse controls. The increased detection frequency among asthmatic participants was more than double that observed in normal individuals, whether judged by number of samples positive (6.4 versus $2.3 \%$, respectively), or by number of participants positive at least once during the study (22 versus $9 \%$, respectively).

The present authors elected to use PCR as the method of detection due to molecular methods of detection being more sensitive than culture in genito-urinary and respiratory chlamydophilal infections $[23,24]$ and because PCR of NA is clearly the method of choice for the detection of upper respiratory infectious agents [2, 3]. The current authors developed a specific RT-PCR for $C$. pneumoniae to detect mRNA coding for the MOMP gene, allowing us to detect replicating organisms. The current authors also elected to use regular sampling every 2 weeks over a 3-month period to determine whether positive detections were short term or chronic. Finally, the use of spouse pairs minimised any differences between groups in terms of exposure to infectious agents.

To the best of the authors' knowledge, the present study is the first to demonstrate a higher frequency of $C$. pneumoniae detection in mild-to-moderate asthmatic participants compared with control participants using direct detection methods. It is possible that the study design employing spouse pairs, among who close proximity might favour higher rates of transmission, may have actually underestimated the degree of increased frequency of detection in asthmatic subjects. Several studies have shown a higher prevalence of antibodies to $C$. pneumoniae in asthmatic compared to normal control subjects [11, 25-27], and one study has shown a relationship to disease severity [12]. These studies have not been able to differentiate acute from chronic infection, although several have hypothesised the presence of chronic $C$. pneumoniae infection. The results of the present study demonstrate that, of detections in which the duration was defined, only one detection could have been of $>28$ days duration. Of the two detections in which duration could not be defined, one ended and the other began during the study. Therefore, it is unlikely that these were chronic infections. The present study has, therefore, been unable to demonstrate the presence of true chronic upper respiratory infections with C. pneumoniae in asthma. In contrast, the current study has demonstrated an increased frequency of detection of upper

Clinical samples

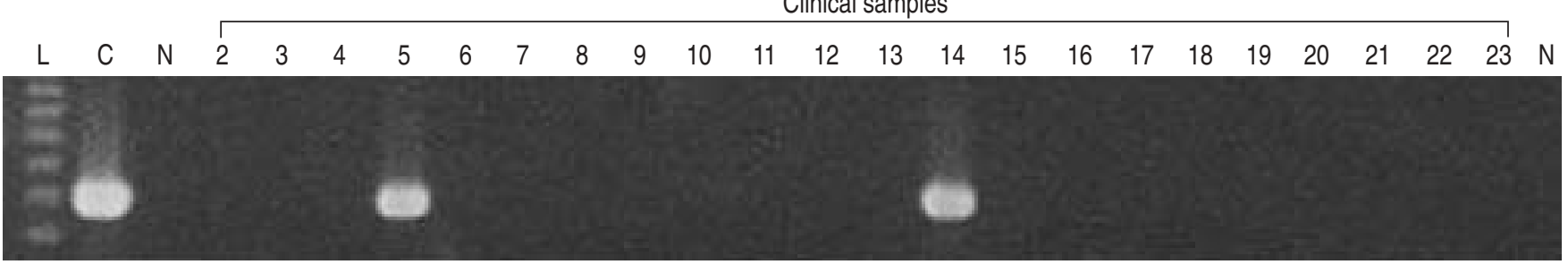

Fig. 2.-RT-PCR detection of Chlamydophila pneumoniae in nasal aspirate samples. L: DNA sizing ladder; C: positive control C. pneumoniae DNA; N: negative control samples. Lanes 2-23 are clinical samples. Lanes C, 5 and 14 are positive for $C$. pneumoniae with clear PCR products of correct size; all others are clearly negative. 
respiratory $C$. pneumoniae detections of short duration in asthma.

The present authors were unable to determine with certainty whether the detected mRNA is a marker for acute infection, reactivation, colonisation or chronic infection. The belief of the current authors is that the PCR-positive detections were detecting very low bacterial loads, hence, the need for a nested PCR to detect them. Therefore, it is possible that these detections relate to short-term colonisation or low-grade reactivation, rather than acute or chronic infections. These data may, therefore, suggest that the previously reported serological data may relate to shortterm colonisation or low-grade reactivation, as opposed to chronic infections. It is interesting to note that all three of the participants with infections of longer duration were asthmatic.

A possible explanation for the above findings would be the presence of impaired immune responses to $C$. pneumoniae infections in asthma. There is no published data on immune responses to $C$. pneumoniae infections in asthma, but the present authors have recently reported impaired antiviral immune responses to rhinoviruses in asthmatic compared to normal subjects [18]. The impaired immune response included deficient production of IFN- $\gamma$ in response to rhinovirus. IFN$\gamma$ is an antiviral cytokine, which is also known to be important in immune responses to $C$. pneumoniae infections $[19,20]$; it is, therefore, possible that impaired production of IFN- $\gamma$ in response to $C$. pneumoniae infections in asthma plays an important role in the increased detection frequency observed in the present study.

An alternative explanation for the previously reported serological findings is that chronic $C$. pneumoniae infections may occur in the lower but not upper respiratory tract in asthma. It was elected to study upper respiratory tract samples in the present study as the authors believed large numbers of participants and samples would be required to address the hypothesis, and obtaining lower respiratory tract specimens involves more invasive procedures, such bronchoscopy or sputum induction. KUOPPA et al. [28] have demonstrated that, with acute infection, the detection rate with a PCR assay for C. pneumoniae is higher from sputum than for throat swabs or nasopharyngeal aspirates. Such studies are now required to investigate whether the present findings reflect the position in the lower respiratory tract.

A possible confounding factor in the present study is the use of inhaled steroids by asthmatic participants. Therefore, the current authors examined whether there was any association between the use of inhaled steroids and the presence of one or more $C$. pneumoniae infections within the asthmatic group. Although BLACK et al. [12] reported that the use of high-dose inhaled steroids was associated with an increase of $>70 \%$ in the titre of $\operatorname{IgA}$ and $\mathrm{IgG}$ antibodies, it was not possible to detect a significant influence of inhaled steroid use on the frequency of detection of $C$. pneumoniae. However, it is possible that the failure to detect such a relationship resulted from lack of power.

In conclusion, the present authors have demonstrated an increased frequency of detection of $C$. pneumoniae infections of $>2$-fold among asthmatic participants compared with normal control participants using RT-PCR in NA from participants matched as closely as possible for exposure to infectious agents. The present authors were unable to find evidence of chronic infection, but detections among asthmatic participants may have been of longer duration than among normal participants.

Further studies are required to determine whether these results are applicable to the asthmatic lower airway and, if so, whether Chlamydophila pneumoniae plays an important role in disease pathogenesis.
Acknowledgements. The authors would like to thank J.L. Low for performing the statistical analysis.

\section{References}

1. The International Study of Asthma and Allergies in Childhood (ISAAC) Steering Committee 1998. Worldwide variation in prevalence of symptoms of asthma, allergic rhinoconjunctivitis and atopic eczema: ISAAC. Lancet 1998; 351: 1225-1232.

2. Johnston SL, Pattemore PK, Sanderson G, et al. Community study of role of viral infections in exacerbations of asthma in 9-11 year old children. BMJ 1995; 310: 1225-1228.

3. Nicholson KG, Kent J, Ireland DC. Respiratory viruses and exacerbations of asthma in adults. BMJ 1993; 307: 982-986.

4. Grayston JT, Kuo C-C, Wang S-P, Altman J. A new Chlamydia psittaci strain, TWAR, isolated in acute respiratory tract infections. New Engl J Med 1986; 315: 161-168.

5. Saikku P. The epidemiology and significance of Chlamydia pneumoniae. J Infect 1992; 25: 27-34.

6. Hammerschlag MR, Chirgwin K, Roblin PM, et al. Persistent infection with Chlamydia pneumoniae following acute respiratory illness. Clin Infect Dis 1992; 14: 178-182.

7. Hyman CL, Augenbraun MH, Roblin PM, Schachter J, Hammerschlag MR. Asymptomatic respiratory infection with Chlamydia pneumoniae TWAR. J Clin Microbiol 1991; 29: 2082-2083.

8. Grayston JT, Campbell LA, Kuo C-C, et al. A new respiratory tract pathogen: Chlamydia pneumoniae strain TWAR. J Infect Dis 1990; 161: 618-625.

9. Hahn DL, Dodge RW, Glubjatnikov R. Association of Chlamydia pneumoniae (strain TWAR) infection with wheezing, asthmatic bronchitis and adult onset asthma. JAMA 1991; 266: 225-230.

10. Emre U, Roblin PM, Gelling M, et al. The association of Chlamydia pneumoniae infection and reactive airway disease in children. Arch Pediatr Adolesc Med 1994; 148: 727-732.

11. Cook PJ, Davies P, Tunnicliffe W, Ayres JG, Honeybourne D, Wise R. Chlamydia pneumoniae and asthma. Thorax 1998; 53: 254-259.

12. Black PN, Scicchitano R, Jenkins CR, et al. Serological evidence of infection with Chlamydia pneumoniae is related to the severity of asthma. Eur Respir J 2000; 15: 254-259.

13. ten Brinke A, van Dissel JT, Sterk PJ, Zwinderman AH, Rabe KF, Bel EH. Persistent airflow limitation in adultonset nonatopic asthma is associated with serologic evidence of Chlamydia pneumoniae infection. J Allergy Clin Immunol 2001; 107: 449-454.

14. Black PN, Blasi F, Jenkins CR, et al. Trial of roxithromycin in subjects with asthma and serological evidence of infection with Chlamydia pneumoniae. Am J Respir Crit Care Med 2001; 164: 536-541.

15. Johnston SL. Is Chlamydia pneumoniae important in asthma? The first controlled trial of therapy leaves the question unanswered. Am J Respir Crit Care Med 2001; 164: 513-514.

16. Cunningham AL, Johnston SL, Julious SA, Lampe FC, Ward ME. Chronic Chlamydia pneumoniae infection and asthma exacerbation in children. Eur Respir J 1998; 11: 345 349 .

17. Corne JM, Marshall C, Smith S, et al. Frequency, severity, and duration of rhinovirus infections in asthmatic and nonasthmatic individuals: a longitudinal cohort study. Lancet 2002; 359: 831-834.

18. Papadopoulos NG, Stanciu LA, Papi A, Holgate ST, Johnston SL. A defective type 1 response to rhinovirus in atopic asthma. Thorax 2002; 57: 328-332.

19. Wizel B, Starcher BC, Samten B, et al. Multiple Chlamydia pneumoniae antigens prime $\mathrm{CD} 8+\mathrm{Tc} 1$ responses that inhibit 
intracellular growth of this vacuolar pathogen. $J$ Immunol 2002; 169: 2524-2535.

20. Rottenberg ME, Gigliotti Rothfuchs A, Gigliotti D, et al. Regulation and role of IFN-gamma in the innate resistance to infection with Chlamydia pneumoniae. J Immunol 2000; 164: 4812-4818.

21. Burney PG, Laitinen LA, Pedrizet S, et al. Validity and repeatability of the IUALDT (1984) bronchial symptoms questionnaire: an international comparison. Eur Respir $J$ 1989; 2: 940-945.

22. Hahn DL, Bukstein D, Luskin A, Zeitz H. Evidence for Chlamydia pneumoniae infection in steroid-dependent asthma. Ann Allergy Asthma Immunol 1998; 80: 45-49.

23. Chernesky MA, Lee H, Schachter J, et al. Diagnosis of Chlamydia trachomatis urethral infection in symptomatic and asymptomatic men by testing first-void urine in a ligase chain reaction assay. J Infect Dis 1994; 170: $1308-1311$.
24. Gaydos CA, Eiden JJ, Oldach D, et al. Diagnosis of Chlamydia pneumoniae infection in patients with community-acquired pneumonia by polymerase chain reaction enzyme immunoassay. Clin Infect Dis 1994; 19: 157-160.

25. Gencay M, Rudiger JJ, Tamm M, Soler M, Perruchoud AP, Roth M. Increased frequency of Chlamydia pneumoniae antibodies in patients with asthma. Am J Respir Crit Care Med 2001; 163: 1097-1100.

26. Von Hertzen L, Toyryla M, Gimishanov A, et al. Asthma, atopy and Chlamydia pneumoniae antibodies in adults. Clin Exp Allergy 1999; 29: 522-528.

27. Von Hertzen L, Vasankari T, Liippo K, Wahlstrom E, Puolakkainen M. Chlamydia pneumoniae and severity of asthma. Scand J Infect Dis 2002; 34: 22-27.

28. Kuoppa Y, Bowan J, Scott L, Kumlin U, Eriksson I, Allard A. Quantitative detection of respiratory Chlamydia pneumoniae infection by real time PCR. J Clin Microbiol 2002; 40: 2273-2274. 